\title{
Influencia de la dinámica familiar percibida en el proyecto de vida en escolares de una institución educativa de Lima
}

\author{
Miguel Barboza-Palomino \\ Universidad Privada del Norte - Trujillo - Peru \\ Indhira Moori \\ Universidad Inca Garcilaso de la Vega - Lima - Peru \\ Stefany Zárate \\ Universidad Inca Garcilaso de la Vega - Lima - Peru \\ Ailyn López \\ Universidad Inca Garcilaso de la Vega - Lima - Peru \\ Kelly Muñoz \\ Universidad Inca Garcilaso de la Vega - Lima - Peru \\ Solange Ramos \\ Universidad Inca Garcilaso de la Vega - Lima - Peru
}

\section{Resumen}

La investigación tuvo como objetivo analizar la influencia de la dinámica familiar percibida en la planeación del proyecto de vida en escolares de 4to año de secundaria de ambos sexos entre 14 a 18 años de una institución educativa del distrito de San Juan de Lurigancho, Lima. Se siguió metodología cualitativa, utilizando la técnica de la entrevista cualitativa que se aplicó a un total de 12 personas. Por otra parte, también se ejecutaron dos grupos focales integrados por siete participantes cada uno. La información recabada fue analizada empleando elementos de la teoría fundamentada y axiomas de conjuntos, empleándose como apoyo el software para el análisis cualitativo de datos Atlas.ti, v. 7.5. Los resultados evidencian que los participantes perciben que factores de la dinámica familiar como el ambiente familiar, la comunicación familiar y tipo de estructura familiar influyen en la construcción de su proyecto de vida.

Palabras clave: Relaciones familiares; proyecto de vida; escolares.

\section{Influence of family dynamics perceived in the life project in schools of an educational institution of Lima}

\begin{abstract}
The aim of this research was to analyze the influence of perceived family dynamics in the construction of the life project among male and female high school students from an educative institution in San Juan de Lurigancho district in Lima city, with ages ranging from 14 to 18 years. To accomplish this goal the study followed a qualitative approach with the application of qualitative interviews to 12 participants and two focus groups formed by seven participants each, as methods of data gathering. The collected information was analysed using elements of grounded theory and axiomatic set theory, with the support of the data analysis package Atlas.ti v. 7.5. Results show that participants perceive that some factors of the family dynamic, such as family environment, family communication, and type of family structure, influence the construction of their life project.
\end{abstract}

Keywords: Family relations; life project; schoolchildren.

\section{Influência da dinâmica familiar percebida no projeto de vida de alunos de uma escola de Lima}

\section{Resumo}

A pesquisa teve como objetivo analisar a influência da dinâmica familiar percebida no planejamento do projeto de vida em estudantes do $4^{\circ}$ ano da escola secundária de ambos os sexos entre 14 e 18 anos de uma instituição de ensino no distrito de San Juan de Lurigancho, Lima. Seguiu-se a metodologia qualitativa com a utilização da técnica de entrevista qualitativa aplicada a um total de 12 pessoas, divididas emdois grupos focais compostos por sete participantes cada. Os dados obtidos foram analisados por meio de elementos da teoria fundamentada e axiomas de conjuntos, utilizando-se como suporteo software de análise qualitativa de dados Atlas.ti, v. 7.5. Os resultados evidenciam que os participantes percebem que fatores da dinâmica familiar como o ambiente familiar, a comunicação familiar e tipo de estrutura familiar influenciam a construção de seu projeto de vida.

Palavras-chave: Relações familiares; projeto de vida; escolares. 


\section{Introduccion}

La familia constituye el núcleo fundamental de la sociedad, ya que es el escenario en el cual se transmiten las creencias, valores y costumbres que influyen en el desarrollo de la persona y la posterior toma de decisiones frente a diferentes situaciones (Minuchin, 1982; Herrera, 2000). Al tener dicha importancia, ha sido foco de diversos estudios, siendo la dinámica y estructura familiar los aspectos que reciben mayor atención en los últimos años (Machado \& Santos, 2007; Gonzales, 2008; Marques \& Manozzo, 2013).

En relación a la dinámica familiar, Eguiluz (2004) señala que implica todas las relaciones que establecen los miembros de la familia entre sí, abarcando el vínculo que construyen los padres, la configuración familiar y el desarrollo de los hijos. De igual forma, Arce (1995) menciona que la dinámica familiar está influenciada por dos indicadores: la comunicación y afectividad, si estos aspectos se encuentran deteriorados se afecta los anhelos de realización personal, autonomía y la asignación de roles, sobre todo de hijos adolescentes. Es así que, diversas investigaciones reportan que una dinámica familiar disfuncional induce a miembros adolescentes a formar parte de pandillas (Gonçalves \& Garcia, 2012), el consumo de alcohol y drogas (Mosqueda-Díaz \& Carvalho, 2011), el intento suicida (Cortés, Aguilar, Suárez, Rodríguez, \& Durán, 2011), el embarazo adolescente (Mendoza \& Subiría, 2013), y un bajo rendimiento académico (Galicia-Moyeda, Sánchez-Velasco, \& Robles-Ojeda, 2013).

Martins y cols. (2007) manifiestan que la dinámica familiar adopta nuevas formas en función a los cambios que experimentan las sociedades, por ende, resulta importante conocer cómo se van configurando las familias con el avance de los años. Así, Puello, Silva y, Silva (2014) mencionan que en los últimos años la estructura familiar se ha visto afectada, incrementándose el número de familias monoparentales, lo cual puede generar repercusiones en la dinámica familiar de grupos que tienen hijos en edad adolescente, siendo más proclives a situaciones de riesgo. Relacionado con lo anterior, el Instituto Nacional de Estadística e Informática (INEI) (2013) refiere que la situación familiar actual en el Perú se caracteriza por un alto índice de familias disfuncionales. Además de lo previamente manifestado, respecto a la influencia de la dinámica familiar disfuncional en distintos aspectos, Gimeno (1999) añade que ésta también puede generar dificultades en sus integrantes adolescentes para proyectar metas a lo largo del tiempo.

Tal como se ha expuesto, la dinámica familiar suele ser vulnerable en familias que tienen miembros en etapa adolescente, debido a que es un periodo que se caracteriza por cambios drásticos en el desarrollo físico, mental, emocional y social (Rodrigo \& cols., 2004). Así también, es importante considerar que el desarrollo de los adolescentes está influenciado por las condiciones económicas, políticas, sociales y culturales del entorno en el cual viven. En ese sentido, el ambiente educativo de los participantes del estudio, se caracteriza por exhibir serias deficiencias, que se acrecentaron producto de la crisis económica y violencia política que experimentó el Perú en la década de los 80 del siglo pasado (León \& Sugimaru, 2013). Asimismo, a pesar de las cifras que indican un crecimiento económico importante en los últimos años, el boom de la economía peruana no se ha reflejado en la atención y mejora de la calidad del servicio educativo por parte del Estado (Caycho \& Barboza-Palomino, 2016).

El sector educativo peruano recibe una partida presupuestal inferior a la de la mayoría de países de América Latina, que se traduce en profesores que reciben remuneraciones salariales que no corresponden a las actividades que desarrollan, a ello se suma la deficiencia de los materiales e infraestructura educativa (Rivero, 2005; Chuquilin, 2011). De igual forma, los adolescentes están expuestos a una serie de problemas sociales que suelen agudizarse en el escenario peruano, tales como el consumo de alcohol, drogas, violencia escolar, pandillas escolares, entre otros (Caycho \& Barboza-Palomino, 2016). Se agrega la desarticulación existente entre los contenidos que se desarrollan en la escuela y las necesidades reales (León \& Sugimaru, 2013).

Es en la adolescencia que se da la búsqueda de una dirección a futuro, lo cual deviene en la construcción de un proyecto de vida, el cual está influenciado por variables personales y circunstancias que rodean al adolescente (Flores, 2008).Es en este punto, que Wallerstein y Lewis (2004) mencionan que la ayuda otorgada en las relaciones entre padres e hijos respecto a la toma de decisiones es de vital importancia, agregan, que los hijos provenientes de familias disfuncionales no mantienen una comunicación cordial y fluida con sus padres acerca de sus proyectos a futuro, a diferencia de los hijos que provienen de familias que exhiben una adecuada dinámica familiar.

Desarrollar un proyecto de vida es importante para el adolescente, ya que le permite plantear metas que desea alcanzar, así también, confeccionar un plan a futuro constituye un factor protector ante situaciones de riesgo, pues se busca que el adolescente proyecte metas orientadas a lograr su bienestar psicosocial (Orcasita \& Uribe, 2010; Páramo, Straniero, García, Torrecilla, \& Escalante, 2012). Lograr lo último mencionado, será posible en la medida que se garanticen diversos aspectos, entre ellos, una adecuada dinámica familiar, que le proporcione al adolescente estrategias específicas para responder y adecuarse a las exigencias y expectativas del medio que lo rodea (Arce, 1995; Eguiluz, 2004).

En lo que respecta a investigaciones previas que hayan abordado el tema de la dinámica familiar y el planeamiento del proyecto de vida en adolescentes, en el contexto internacional, se encuentra a Castro y Díaz (2002) quienes buscaron conocer el proyecto de vida de estudiantes de educación secundaria de Argentina y España, para lo cual emplearon la metodología de Little. Los participantes del estudio fueron 816 estudiantes entre varones y mujeres de institutos de educación secundaria. Los resultados mostraron que un $21 \%$ de los estudiantes han desarrollado en gran medida sus objetivos de vida en el momento actual, logrando así un alto nivel de autosatisfacción, mientras que un $17 \%$ del grupo percibe que han desarrollado muy poco sus objetivos de vida en el momento presente y no esperan 
desarrollarlos en un futuro, por ende, tienen un bajo nivel de autosatisfacción en relación con sus logros. Se concluye que sólo una quinta parte de los participantes logró desarrollar sus objetivos de vida, asimismo, se visualiza la relación que guardan con la autosatisfacción.

A lo mencionado, Peres y Monterio (2003), en función a resultados de su investigación ejecutada con adolescentes atendidos en una unidad básica de salud en Sao Paulo (Brasil), sostienen que los adolescentes definen un proyecto de vida, sin embargo, identifican condiciones socioeconómicas que dificultarían lograrlas metas trazadas. De igual forma, consideran a la relación familiar como un factor protector e importante para plantear metas a futuro. No obstante, reconocen que a veces no se genera un clima adecuado que permita hablar de temas relacionados con el consumo de drogas, embarazo o enamoramiento.

Así también, Machado y Santos (2007) tuvieron por objetivo identificar los puntos positivos y negativos de la dinámica familiar en relación a los planes futuros que identifican adolescentes consumidores y no consumidores de sustancias psicoactivas. Aplicaron un cuestionario de auto-respuesta a 568 estudiantes de ambos sexos entre 14 a 20 años que cursaban estudios en escuelas públicas y privadas de la ciudad de Sao Carlos (Brasil). Los resultados indicaron que tanto adolescentes consumidores y no consumidores de sustancias psicoactivas identifican a la dinámica familiar como un factor crucial en relación a la planeación del futuro, tal es así que, una dinámica familiar positiva garantizará una adecuada planeación de metas futuras, a diferencia de una dinámica familiar negativa.

Asimismo, Marcelino, Catão, y Lima (2009) ejecutaron un estudio con el objetivo de comparar representaciones sociales de adolescentes de escuelas públicas y privadas en relación a su proyecto de vida. Emplearon para la recolección de datos una encuesta sociodemográfica y realizaron entrevistas semi estructuradas a dos grupos de 20 estudiantes brasileros (uno perteneciente a una escuela privada y el otro a una escuela pública). Entre los principales hallazgos, reportan que los estudiantes de escuelas públicas reconocen dificultades en la construcción de su proyecto de vida a diferencia de los participantes de escuelas privadas. Además, las dificultadas identificadas están mayoritariamente asociadas a variables personales, no obstante, resalta la presión social que puede estar dada por diferentes grupos, entre ellos, la familia.

Por su parte, Ruiz (2011) realizó un estudio con el objetivo de evaluar e intervenir en el proceso de toma de decisiones en la construcción del plan de vida. Trabajó con una muestra de diez estudiantes, cinco varones y cinco mujeres, entre 15 a 18 años de un colegio de Cundinamarca (Colombia). Para la recolección de datos utilizó la técnica de los relatos autobiográficos, lo cual facilitó conocer su contexto familiar, situación familiar, situación económica, posibilidades y limitaciones para su desarrollo a futuro. Los estudiantes identificaron en sus familias buenos y malos tratos por parte de sus cuidadores, y en algunos casos abuso. Los diez participantes planean cambios en su núcleo familiar, desde el acercamiento a través de la comunicación con sus padres y hermanos e integrarse en las actividades familiares como una forma de mejorar las relaciones familiares. También identificaron que la manera de resolver sus problemas económicos es culminando sus estudios, tanto secundarios como superiores, para lograr conseguir un empleo estable.

En lo que respecta al escenario peruano, no se encuentran estudios previos acerca del tema de investigación, sin embargo, resaltan documentos institucionales de organizaciones públicas y privadas que han desarrollado actividades orientadas a informar, sensibilizar y promover en el adolescente la planeación de un proyecto de vida (Ministerio de Educación [MINEDU], 2011), siendo el distrito de San Juan de Lurigancho, área de mayor población en Lima capital y lugar de residencia de los participantes del estudio, uno de los ámbitos educativos que mayor atención recibe (Parodi-Carro, 2014). Resulta pertinente señalar que estas actividades están relacionadas generalmente a orientar a los estudiantes a continuar estudios de educación superior, respecto a lo cual, se ha evidenciado queen su decisión influyen variables como la educación e historia educativa de los padres (Castro, Yamada, \& Arias, 2011). No obstante, esta concepción de proyecto de vida resulta limitada, puesto que, en el Perú se ha demostrado que sólo uno de cada cinco adolescentes deciden continuar estudios superiores, por lo que se está dejando de lado el estudio de adolescentes que no plantean la posibilidad de continuar su formación superior y se inclinan a desarrollar inmediatamente actividades laborales, así como, a los adolescentes que provienen generalmente de familias monoparentales y que deciden no estudiar ni trabajar (León \& Sugimaru, 2013).

De acuerdo a lo expuesto, la presente investigación se enfocó en indagar la relación entre la dinámica familiar percibida y el proyecto de vida en escolares de una institución educativa pública del distrito de San Juan de Lurigancho. Se plantearon los siguientes objetivos de investigación:

(i) Analizar la influencia de la dinámica familiar percibida en la elaboración del proyecto de vida en escolares de una institución educativa de Lima.

(ii) Interpretar la valoración que atribuyen a la dinámica familiar escolares de una institución educativa de Lima.

(iii) Interpretar el significado que le atribuyen al proyecto de vida escolares de una institución educativa de Lima.

La investigación comunica a nivel de resultados la interpretación que elaboran los participantes del estudio de la dinámica familiar y la planeación del proyecto de vida, así como, analiza la percepción de influencia que construyen entre los conceptos mencionados.

\section{Método}


La investigación siguió metodología cualitativa, ya que se enmarca dentro de la lógica epistemológica subjetivista (Montero \& León, 2007). Se adoptó el diseño fenomenológico, puesto que, se buscó conocer cómo experimentan desde su propia perspectiva los participantes el tema de estudio (Creswell, 1998; Toledo-Nickels, 2009).

\section{Participantes}

La presente investigación se llevó a cabo con la participación de 26 escolares adolescentes de ambos sexos (13 varones; 13 mujeres) del 4to. año de educación secundaria (población total de 40 estudiantes), cuyas edades estaban en el rango de edad de 14 a 18 años del turno tarde de la I.E. N 0090 Daniel Alcides Carrión, ubicado en la zona de Campoy - San Juan de Lurigancho, Lima.

La selección de los participantes se hizo empleando el tipo de muestreo intencional teórico (Ruiz, 2012), pues el objetivo era construir una teoría que permita explicar el tema de investigación en la población de estudio. La cantidad tope de participantes en el estudio se estableció utilizando el criterio de saturación teórica de la teoría fundamentada (Corbin \& Strauss, 2015).

\section{Técnicas y materiales}

En el proceso de la recolección de información se utilizó la técnica de la entrevista cualitativa, la cual fue aplicada a 12 personas (seis varones y seis mujeres), por otra parte también se realizaron dos grupos focales integrados por siete estudiantes de ambos sexos (participaron 14 personas en los dos grupos focales, siete varones y siete mujeres).

Para la aplicación de cada una de las técnicas se elaboró y validó una guía temática de entrevista y grupo focal, la cual estuvo constituida por las siguientes áreas de investigación: Estructura y organización familiar, relaciones familiares, funcionalidad familiar, ambiente familiar, afectividad familiar, plan de vida, percepción de sí mismo, percepción de su futuro y planteamiento de metas. El desarrollo de cada una de las técnicas expuestas estuvo supeditado previamente a la presentación y firma del consentimiento informado por cada uno de participantes del estudio.

\section{Procedimiento}

Seleccionadas las técnicas para la recolección de información, se elaboró una guía temática que comprendió las mismas áreas de investigación tanto para la entrevista cualitativa como para el grupo focal, ello, al compartir la misma naturaleza las técnicas seleccionadas (Rubin \& Rubin, 2012; Barbour, 2007). La guía temática fue validada a través de la ejecución de una prueba piloto (una entrevista a un participante con las mismas características de la muestra), empleándose el criterio de calidad de confirmación por los participantes (Creswell, 2013). La información recabada permitió estructurar la guía temática, así como también facilitó la construcción de un libro de códigos para el análisis de datos.

Las entrevistas cualitativas se desarrollaron en una sola sesión de 45 minutos aproximadamente, por su parte los grupos focales se ejecutaron en un tiempo de una hora y 30 minutos aproximadamente, efectuándose el registro de la información empleando grabadoras de audio, video y cuadernos de campo. La información recolectada fue transcrita y luego analizada empleando los elementos de la teoría fundamentada (Corbin \& Strauss, 2015) y axiomas de conjuntos, de igual forma, se utilizó como apoyo el software para el análisis cualitativo de datos Atlas.ti v.7.5.

El proceso de análisis de información se inició realizando la familiarización con los datos recolectados, posterior a ello, se comenzó a seleccionar segmentos de información a los cuales se les fue asignado los códigos necesarios, empleando para ello un libro de códigos elaborado a partir de la entrevista piloto y conceptos que se desprendieron de la revisión de literatura en relación al tema de investigación. Finalizado el proceso de codificación, se inició la fase de vincular los códigos y creación de categorías (códigos con un mayor nivel de jerarquía conceptual), utilizando para ello las propiedades matemáticas de los axiomas de Zermelo-Fraenkel (Anacona, Arboleda, \& Pérez-Fernández, 2014) permitiendo la generación de visualizaciones teorizadas.

El presente estudio para garantizar su rigurosidad científica siguió los siguientes criterios de calidad: Triangulación de métodos y triangulación de investigadores (Creswell, 2013).

\section{Resultados}

El análisis de los datospermite afirmar quelos participantes conceptualizan a la dinámica familiar como un factor que influye en la construcción de un proyecto de vida, estando constituido por cinco componentes: el ambiente familiar, la funcionalidadfamiliar, la comunicación, la afectividad familiar y estructura familiar.

En relación al ambiente familiar, los escolares la definen como el clima social que se vive en la familia, asimismo, las opiniones se dividen en torno a la satisfacción e insatisfacción que les genera su ambiente familiar cotidiano, por otra parte, coinciden en señalar que lo ideal es la construcción de un ambiente familiar adecuado, cuya responsabilidad, a juicio de los participantes, recae en la figura de los padres.

Por su parte, la funcionalidad familiar es caracterizada como la forma en la cual la familia se relaciona y actúa frente a diferentes situaciones, identificando que dicho aspecto puede ser adecuado o inadecuado, desarrollándose la primera en la medida que se definan responsabilidades y normas claras. Además, perciben que la mayoría de familias peruanas carecen de normativas adecuadas en su interior, lo cual dificulta su funcionamiento, generando problemas como: hijos desobedientes, falta de respeto a la autoridad, escasa comunicación familiar, desunión familiar. 


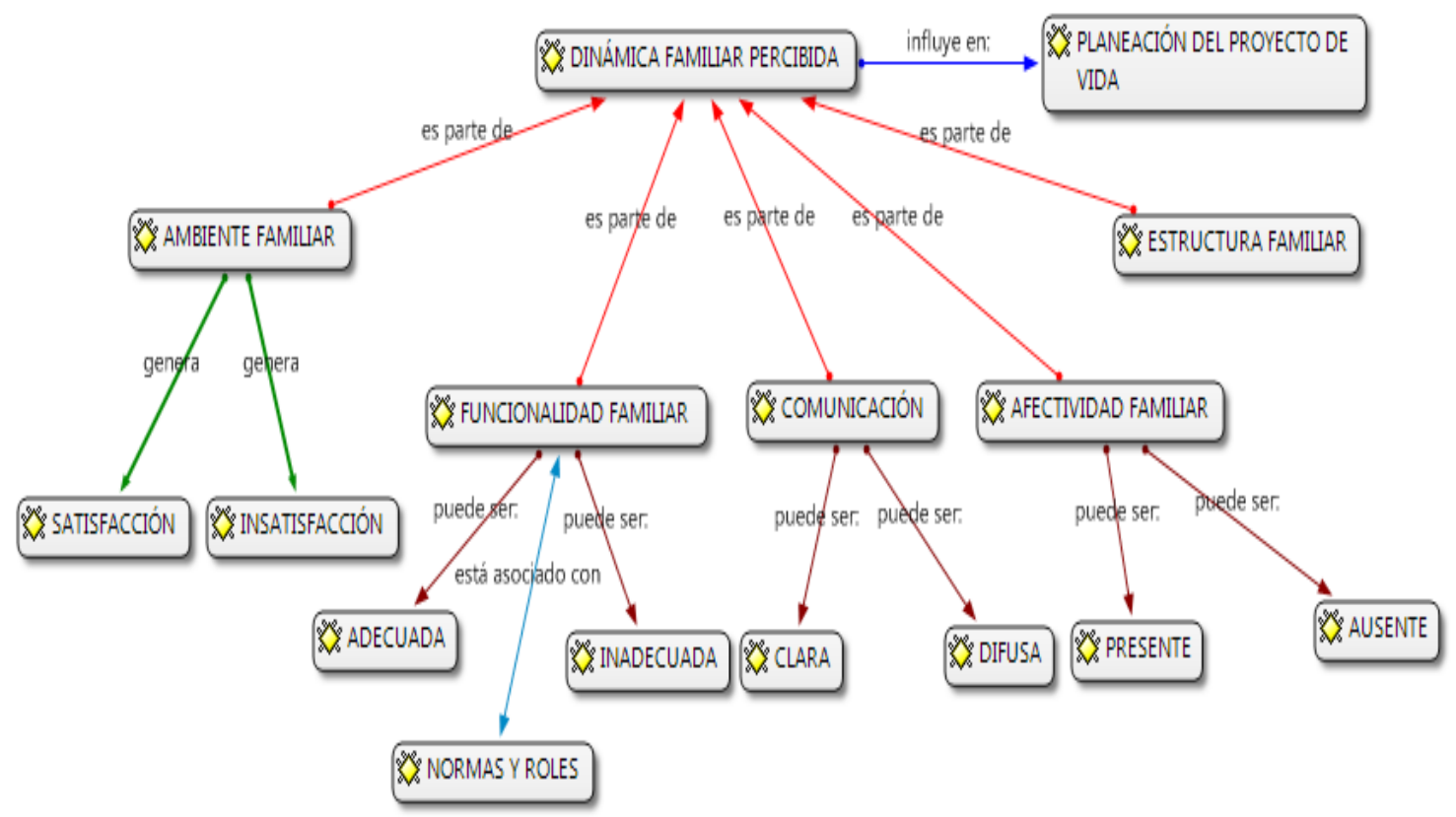

Figura 1. Componentes y características de la dinámica familiar.

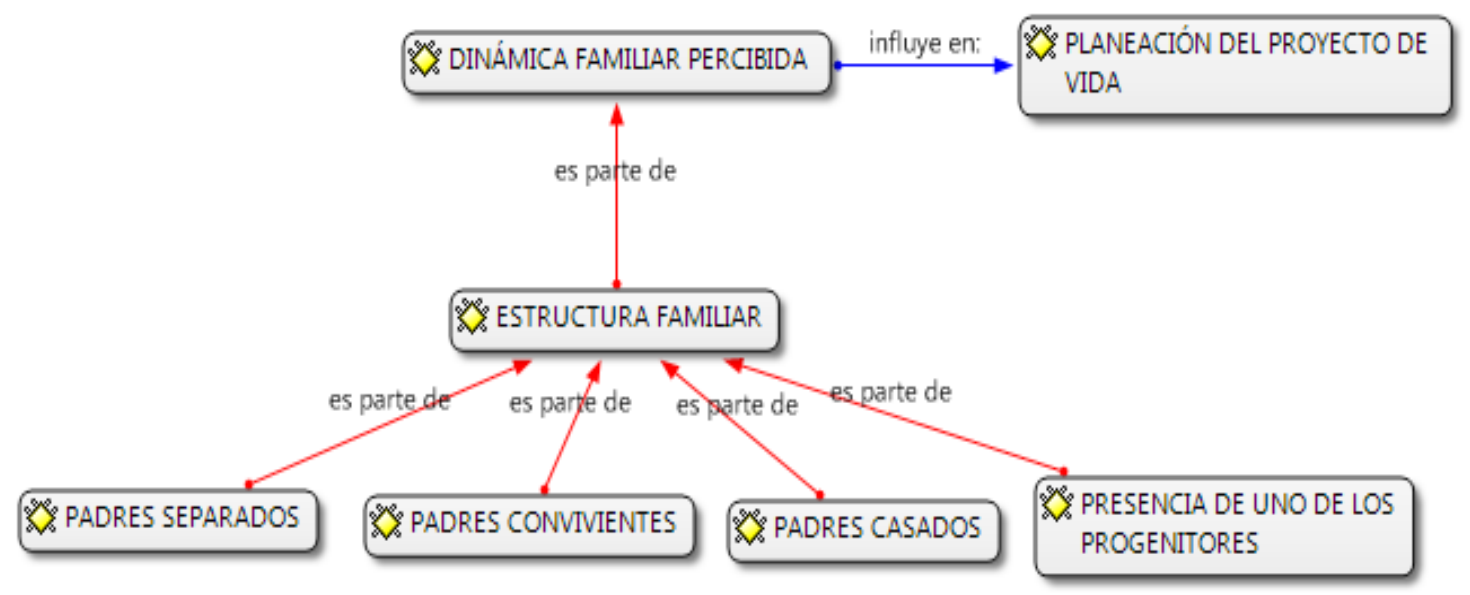

Figura 2. Tipos de estructura familiar que identifican escolares peruanos.

En lo que respecta a la comunicación, la visualizan como la frecuencia y claridad en el intercambio de mensajes entre integrantes de la familia, señalando la mayoría de participantes que en su familia se presenta una comunicación difusa, por su parte, el resto de los integrantes afirma que en su entorno familiar se experimenta una comunicación clara. Asimismo, añaden que una comunicación clara se construye en la medida que exista receptividad por parte de los padres. Por otro lado, definen a la afectividad familiar como la presencia de expresiones emocionales de afecto en la relación padres-hijos, señalando la mayoría de participantes que ésta se encuentra ausente en su familia.

Los participantes señalan a la estructura familiar como el componente resaltante de la dinámica familiar, siendo definida como la forma en que está organizada la familia a partir del tipo de vínculo que construyen los padres, así como la presencia-ausencia de los mismos. Los escolares manifiestan que sus familias se caracterizan por las siguientes composiciones: padres separados, padres casados, padres convivientes, presencia de uno de los progenitores.

Cada uno de los tipos de estructura familiar identificado, a juicio de los escolares, no guarda una relación directa con la calidad de dinámica familiar que se construye, pudiendo presentarse, como lo afirman, familias de padres casados que promueven una inadecuada dinámica familiar, o familias con la presencia de uno de los progenitores que fomentan una adecuada dinámica familiar. Sin embargo, señalan que en la medida que se dé la presencia de ambos padres existe una mayor probabilidad de generar una adecuada dinámica familiar, pues, manifiestan que resulta necesario el apoyo conjunto para tomar decisiones respecto a asuntos que involucran aspectos del desarrollo personal.

En relación al proyecto de vida, los participantes del estudio interpretan que su construcción está influenciada 
por la calidad de dinámica familiar que se construya, definiéndola como una acción importante y crucial de proyectar metas a cumplir en el futuro, la cual no está relacionado sólo con la decisión de seguir una carrera profesional, ya que, algunos participantes afirman el interés de desarrollar actividades deportivas y artísticas. Sin embargo, la mayoría de escolares elaboran una visión a futuro ligada a un desarrollo técnico u profesional en un instituto o universidad.

Las metas que los estudiantes plantean están condicionadas a 3 factores: expectativa económica, expectativas familiares y percepción de sí mismo. La expectativa económica constituye una evaluación que realizan los escolares acerca de las posibilidades de mantención/apoyo económico en educación de parte de los padres, reconociendo que algunos planes no son viables, al presentar dificultades de solvencia económica.

Por su parte, las expectativas familiares son caracterizadas como los anhelos profesionales u ocupacionales que los padres construyen acerca de los hijos, los cuales influyen de forma directa en la toma de una decisión vocacional en la medida que en el escenario familiar exista una adecuada comunicación, así también, por la funcionalidad familiar, destacando los escolares que las figuras parentales que asumen roles autoritarios tienden a influir en lo que sus hijos deciden estudiar o hacer a futuro.

Finalmente la percepción de sí mismo, es conceptualizada como la visión personal de fortalezas y debilidades que ayudarán o dificultarán el logro de metas planteadas por el adolescente. Al respecto, los participantes añaden que una percepción positiva es construida por diferentes factores, entre ellos, resaltan el papel que desempeña una adecuada dinámica familiar. No obstante, afirman que en familias donde existe una inadecuada dinámica familiar también los hijos pueden desarrollar una percepción positiva de sí mismo, convirtiéndose ésta en una herramienta personal que le permitirá plantear metas y trabajar por el cumplimiento de las mismas. De igual forma, señalan que la percepción negativa es construida por diversas circunstancias, entre ellos destacan aspectos de la dinámica familiar como un inadecuado ambiente familiar y comunicación familiar difusa.

Los participantes perciben la dinámica familiar como elemento influyente en la elaboración de su proyecto de vida. Los distintos componentes que identifican guardan relación con los factores que intervienen en la planeación del proyecto de vida, sin embargo, cabe afirmar que los escolares manifiestan que la relación no es determinante.

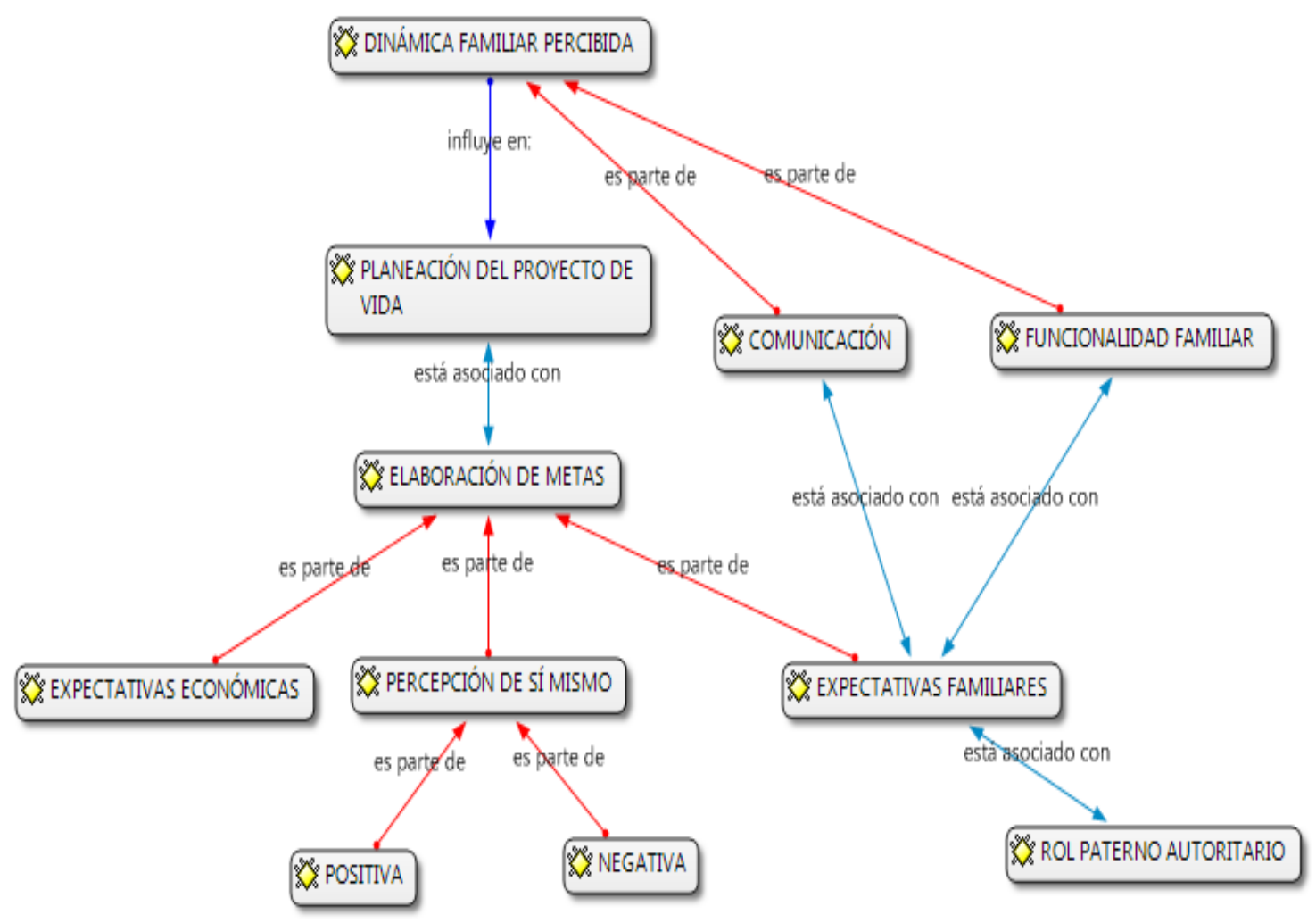

Figura 3. Características de la dinámica familiar que influyen en la planeación de un proyecto de vida. 


\section{Discusión}

La presente investigación buscó analizar cómo influye la dinámica familiar percibida en la planeación del proyecto de vida en escolares de una institución educativa del distrito de San Juan de Lurigancho (Lima), considerando diversos aspectos, entre ellos: la familia como sistema básico de socialización y desarrollo de estrategias para la toma de decisiones, la adolescencia y importancia de la construcción de un proyecto de vida. Cabe indicar que la percepción que elaboran los participantes acerca de su dinámica familiar e influencia en la planeación de metas a futuro está condicionada por los factores económicos, políticos, sociales y culturales del entorno (Rivero, 2005; Chuquilin, 2011; León \& Sugimaru, 2013; Caycho \& Barboza-Palomino, 2016).

Los resultados de la investigación permiten establecer principios desde la perspectiva de los participantes: la existencia de una relación de influencia, no determinante, entre la dinámica familiar percibida y planeación de un proyecto de vida; la conceptualización de percibir una adecuada dinámica familiar como una variable que predice de forma positiva la construcción de un proyecto de vida; la existencia de factores ajenos a la dinámica familiar que pueden incidir en el desarrollo de recursos positivos personales para tomar decisiones respecto al futuro.

En lo que respecta a la conceptualización de la dinámica familiar, los resultados muestran a diferencia de lo señalado por Arce (1995), que ésta no se reduce a la comunicación y afectividad familiar, al contrario, tal cual lo afirma Eguiluz (2004), abarca distintos aspectos, como el ambiente, funcionalidad y estructura familiar. Por otra parte, se añade a lo expuesto por Machado y Santos (2007), que los adolescentes participantes (identificados como no consumidores de sustancias psicoactivas), también reconocen aspectos negativos en los componentes y características de la dinámica familiar. Es así que, efectúan una connotación positiva y negativa: ambiente familiar (adecuado-inadecuado), funcionalidad familiar (adecuada-inadecuada), comunicación (clara-difusa), afectividad familiar (presente, ausente). Lo descrito resulta importante para plantear discusiones conceptuales acerca de la dinámica familiar, además, resulta útil en la orientación de estudios que empleen instrumentos psicométricos dirigidos a medir la dinámica familiar en adolescentes, puesto que, debiesen considerar los componentes y características identificados.

También, los escolares señalan que una dinámica familiar adecuada facilita el desarrollo de estrategias para la toma de decisiones a futuro, lo afirmado es símil a lo mencionado por Wallerstein y Lewis (2004), Machado y Santos (2007), y Peres y Monterio (2003), asimismo, en correspondencia a lo indicado por Gimeno (1999), se identificó que un ambiente familiar hostil constituye un factor que dificulta la planeación de un proyecto de vida. De otro lado, al igual que lo señalado por Martins y cols. (2007), los participantes reconocen que la familia va adoptando nuevas formas en función a los cambios que experimenta la sociedad. Por su parte, de acuerdo a lo que afirma el INEI (2013), se evidencia en la mayoría de escolares, el reconocimientode pertenencia a una familia disfuncional. Además, contrario a lo que manifiesta Ruiz (2011), los participantes no plantean como una necesidad cambiar escenarios familiares identificados como hostiles, ello sí, consideran que lo ideal sería convivir en un ambiente familiar adecuado.

Los participantes del estudio reconocen que la adolescencia es una etapa que se caracteriza por la necesidad de plantear un proyecto de vida, de igual forma, señalan que en dicho periodo experimentan una serie de cambios que generan sentimientos de vulnerabilidad ante diferentes situaciones, influyendo de forma marcada la vivencia personal-familiar de cada adolescente, lo encontrado es análogo a lo que afirman Rodrigo y cols. (2004), Flores (2008), Marcelino y cols. (2009), y Castro y cols.(2011).

Respecto a lo señalado por Castro y Díaz (2002), en la investigación no se encontró que los participantes establezcan relación entre satisfacción y posible cumplimiento de las metas de sus proyectos de vida, sin embargo, sí establecen una relación entre satisfacción y adecuado ambiente familiar. Así también, se contradice lo señalado por Ruiz (2011), ya que los participantes desvinculan la posibilidad de estudiar como una alternativa para solucionar problemas económicos. Se añade que, los escolares elaboran metas ligadas a actividades no relacionadas de forma exclusiva a continuar estudios superiores. Al igual que lo encontrado por Peres y Monteiro(2003), los adolescentes identifican condiciones socioeconómicas que dificultarían alcanzar las metas trazadas.

Por otra parte, los participantes reconocen aspectos relacionados a la construcción del proyecto de vida y cómo influyen distintos factores, entre ellos, la familia. Sin embargo, perciben de forma no significativa las actividades de orientación que se desarrollan en la escuela, por lo que se requiere evaluarse la organización y ejecución de dichas actividades, partiendo desde lo dispuesto por el Estado hasta lo realizado por las instituciones educativas. Cabe resaltar que la concepción de proyecto de vida que prevalece en el escenario peruano está asociado a continuar estudios superiores, estando dirigidas la mayoría de actividades a estudiantes que cumplan con la condición manifiesta, no obstante, la realidad indica que la suma mayoritaria de adolescentes que egresan de las escuela no permanecen en el sistema educativo (León \& Sugimaru, 2013), validándose la necesidad de seguir desarrollando estudios en relación al tema con adolescentes en etapa escolar.

Se rescata la conceptualización favorable de parte de los escolares de los distintos ámbitos vinculados con la dinámica familiar. Lo descrito es una potencialidad, puesto que, el Estado, instituciones y agentes educativos debiesen favorecerse de ésta disposición para desarrollar y consolidar actividades que permitan fomentar dinámicas familiares adecuadas. Se recuerda, que una dinámica familiar adecuada favorece la construcción de un proyecto de vida, que se convierte en un factor protector frente a diferentes situaciones de riesgo (Orcasita \& Uribe, 2010; Mosqueda-Díaz \& Carvalho, 2011; Cortés \& cols., 2011; Páramo \& cols. 2012; Gonçalves \& Garcia, 2012; Mendoza \& Subiría, 2013; Galicia-Moyeda \& cols., 2013). 
Finalmente, se evidencia la escases de información científica acerca del tema de la presente investigación, por lo cual, se requiere a futuro desarrollar estudios que consideren otras variantes. En ese sentido, someter a contraste lo planteado por Puello y cols.(2014), así como lo señalado por el INEI (2013), permitirá, por ejemplo, entender como la estructura familiar o las familias monoparentales, repercuten en la dinámica familiar de grupos con hijos adolescentes. De igual forma, es necesario caracterizar muestras de acuerdo a estratos socioeconómicos (Peres \& Monterio, 2003), tipo de institución de procedencia (Marcelino \& cols., 2009), entre otras variables, pudiendo valorarse diferencias.

\section{Conclusiones}

De acuerdo al análisis de los resultados y su posterior discusión se concluye:

(i) Los participantes del estudio perciben a la dinámica familiar como un factor influyente en la planeación de su proyecto de vida, sin embargo, no establecen una relación determinante.

(ii) Los escolares consideran que una adecuada dinámica familiar predice de forma positiva la planeación del proyecto de vida, señalando que ésta involucra los siguientes componentes: ambiente familiar, funcionalidad familiar, la comunicación, afectividad familiar y estructura familiar.

(iii) Los estudiantes valoran positivamente la planeación de un proyecto de vida, afirmando que implica elaborar metas, las cuales estarán condicionadas por las expectativas económicas, expectativas familiares y percepción de sí mismo.

\section{Referencias}

Anacona, M., Arboleda, L. C., \& Pérez-Fernández, F. J. (2014). On Bourbaki's axiomatic system for set theory. Synthese, 17(191), 4069-4098.

Arce, M. (1995). El proceso de socialización y los roles en la familia. Teoría y metodología para la intervención en familias. San José: Universidad Nacional de Costa Rica.

Barbour, R. (2007). Doing Focus Groups. California: Sage.

Caycho, T. \& Barboza-Palomino, M. (2016). Retos actuales en el escenario educativo peruano: Repensando el rol del psicólogo educativo. Em C. Cornejo \& G. Salas (Orgs.), Desde el aula. Ensayos sobre psicología y educación (pp. 102-107). La Serena: Nueva Mirada Ediciones.

Castro, A. \& Díaz, J. (2002). Objetivos de vida y satisfacción vital en adolescentes españoles y argentinos. Revista Psicothema, 14(1),
112-117.

Castro, F. J., Yamada, G., \& Arias, O. (2011). Higher Education Decisions in Peru: On the Role of Financial Constraints, Skills and Family Background. (Documento de Discusión). Lima: Centro de Investigación de la Universidad Pacífico.

Chuquilin, J. (2011). La educación secundaria en Perú y sus profesores: cambios y continuidades. Revista Educación, 35(2), 95-117.

Corbin, J. \& Strauss, A. (2015). Basics of Qualitative Research. Techniques, and Procedures for Developing Grounded Theory. California: Sage

Cortés, A., Aguilar, J., Suárez, R., Rodríguez, E. \& Durán, J. S. (2011). Factores de riesgo asociados con el intento suicida y criterios sobre lo ocurrido en adolescentes. Revista Cubana de Medicina Integral, 27(1), 33-41,

Creswell, J. W. (1998). Qualitative inquiry and research design. Choosing Among Five Approaches. California: Sage.

Creswell, J. W. (2013). Qualitative inquiry and research design. Choosing Among Five Approaches (Third Edition). California: Sage.

Eguiluz, L. L. (2004). Dinámica de la familia: un enfoque psicológico sistémico. México, D. F.: Editorial Pax.

Flores, M. S. (2008). Resiliencia y proyecto de vida en estudiantes del tercer año de secundaria de la UGEL 03. Tesis para optar el grado académico de Magister en Psicología Educativa, Universidad Nacional Mayor de San Marcos.

Galicia-Moyeda, I. X., Sánchez-Velasco, A., \& Robles-Ojeda, F. J. (2013). Autoeficacia en escolares adolescentes: su relación con la depresión, el rendimiento académico y las relaciones familiares. Anales de psicología, 29(2), 491-500.

Gimeno A. (1999). La familia: el desafío de la diversidad. Barcelona: Ariel.

Gonçalves, J. \& Garcia, A. C. (2012). Violência e fragilidades nas relações familiares: refletindo sobre a situação de adolescentes em conflito com a lei. Estudos de Psicologia, 17(3), 389-395.

Gonzales, A. (2008) La familia. Una mirada desde la Psicología. Revista Electrónica de las Ciencias Médicas en Cienfuegos, 6(1), 4-13.

Herrera, P. (2000) Rol de género y Funcionamiento familiar. Revista Cubana de Medicina General Integral, 16(6) 568-573.

Instituto Nacional de Estadística e Informática (2013). 11 de julio. Día Mundial de la Población. Recuperado: 15 oct. 2015. Disponível:www.inei.gob.pe/media/MenuRecursivo/ 
publicaciones_digitales/Est/Lib1095/libro.pdf

León, J. \& Sugimaru, C. (2013). Entre el estudio y el trabajo: Las decisiones de los jóvenes peruanos después de concluir la educación básica regular. (Avances de investigación. Educación y aprendizaje). Lima: Grupo de Análisis para el Desarrollo, GRADE.

Machado, E. M. \& Santos, M. A. (2007). Opiniões dos adolescentes do ensino médio sobre o relacionamento familiar eseus planos para o futuro. Paidéia, 17(36), 103-114.

Marcelino, M. Q. S., Catão, M. F. F. M., \& Lima, C. M. P. (2009). Social representations of life project among adolescents in high school. Psicologia: Ciência e Profissão, 29(3), 544-557.

Marques, F. \& Manozzo, P. (2013). Aspectos da dinâmica da família com dependência química. Estudos de Psicologia, 18(4), 551-558

Martins, C. S., Ferriani, M. D. G. C., Silva, M. A. I., Zahr, N. R., Arone, K. M. B.,\& De Roque, E. M.S. T. (2007). Family dynamics from the perspective of parents and children involved in domestic violence against children and adolescents. Revista Latino-Americana de Enfermagem, 15(5), 889-894.

Mendoza, W. \& Subiría, G. (2013). El embarazo adolescente en el Perú: Situación actual e implicancias para las políticas públicas. Revista Peruana de Medicina Experimental y Salud Pública, 30(3), 471-479.

Ministerio de Educación [MINEDU] (2011). Orientación vocacional. Cartilla para tutores. Recuperado: 30 oct. 2015. Disponível: www. cpnsrosario.edu.pe/Dpto.\%20Psicopedagogico/Tutoria/Sesiones/ ORIENTACION\%2OVOCACIONAL.pdf

Minuchin (1982). Familias y terapia familiar. Buenos Aires: Gedisa.

Montero, O. \& León, I. (2007). A guide for naming research studies in Psychology. International Journal of Clinical and Health Psychology, 7(3), 847-862.

Mosqueda-Díaz, A. \& Carvalho, M. G. (2011). Factores protectores y de riesgo familiar relacionados al fenómeno de drogas, presentes en familias de adolescentes tempranos de Valparaíso, Chile. Revista Latino-Americana de Enfermagem, 19, 789-795.
Orcasita, L. T. \& Uribe, A. F. (2010). La importancia del apoyo social en el bienestar de los adolescentes. Psychologia: Avances de la Disciplina, 4(2), 69-82.

Páramo, M. A., Straniero, C. M., García, C. S., Torrecilla, N. M., \& Escalante, E. (2012). Bienestar psicológico, estilos de personalidad y objetivos de vida en estudiantes universitarios. Pensamiento Psicológico, 10(1), 7-21.

Parodi-Carro, A. F. (2014). Educación sexual y perfil del adolescente de nivel secundario en San Juan de Lurigancho - Lima. Cuidado y Salud, 1(2), 67-76.

Peres, C. \& Monteiro, M. I. (2003). Projeto de vida de um grupo de adolescentes à luz de Paulo Freire. Revista Latino-Americana de Enfermagem, 11(6), 778-785.

Puello, M., Silva, M., \& Silva, A. (2014). Límites, reglas, comunicación en familia monoparental con hijos adolescentes. Diversitas: Perspectivas en Psicología, 10(2), 225-246.

Rivero, J. (2007). La Educación Peruana: crisis y posibilidades. ProPosições, 16(2), 199-218.

Rodrigo, J., Máiquez, L., García, M., Mendoza, R., Rubio, A., Martínez, A., \& Martín, J. (2004). Relaciones padres-hijos y estilos de vida en la adolescencia. Revista Psicothema,16(2), 203-210.

Rubin, H. J. \& Rubin, I. S. (2012).Qualitative Interviewing. The Art of Hearing Data. California: Sage.

Ruiz, J. (2011). Proyecto de vida, relatos autobiográficos y toma de decisiones. Revista Teoría y Praxis Investigativa, 6(1), 27-34.

Ruiz, J. I. (2012). Metodología de la investigación cualitativa (5ta. Edición). Bilbao: Universidad de Deustuo.

Toledo-Nickels, U. (2009). El programa socio-fenomenológico de investigación. Cinta Moebio, (35), 67-87

Wallerstein, J., \& Lewis, J. (2004). The Unexpected Legacy of Divorce: Report of a 25-Year Study. Psychoanalytic Psychology, 21(3). 


\section{Sobre os autores}

Miguel Barboza-Palomino (mbarbozapalomino@outlook.com.pe)

Universidad Privada del Norte. Candidato a Magister en Psicología Educativa por la Universidad Nacional Mayor de San Marcos. Miembro del Instituto de Investigación Walter Blumenfeld Meyer.

Indhira Moori (indhira2410@gmail.com)

Investigadora de la Universidad Inca Garcilaso de la Vega

Stefany Zárate (stefany.zarate@upch.pe)

Investigadora de la Universidad Inca Garcilaso de la Vega

Ailyn López (aylosa_forever@hotmail.com)

Investigadora de la Universidad Inca Garcilaso de la Vega

Kelly Muñoz (kelly_25abril@hotmail.com)

Investigadora de la Universidad Inca Garcilaso de la Vega

Solange Ramos (solange.ramos13@gmail.com)

Investigadora de la Universidad Inca Garcilaso de la Vega 